\title{
Time- and Spatial-Harmonic Content in Synchronous Electrical Machines
}

\author{
Bert Hannon ${ }^{1}$ Member, IEEE,Peter Sergeant ${ }^{1}$ Member, IEEE,Luc Dupré ${ }^{1}$ Member, IEEE \\ ${ }^{1}$ Dept. EESA, Electrical Energy Laboratory, Ghent University, Valentin Vaerwyckweg 1, 9000 Gent, Belgium
}

\begin{abstract}
The use of power electronics has led to a growing importance of higher time-harmonic content in electrical machines. To gain insight in phenomena related to these higher harmonics, such as noise and losses, a good understanding of the magnetic field's harmonic content is mandatory. Moreover, the development of fast and accurate, harmonic-based, analytical models requires a qualitative knowledge of the machine's time- and spatial-harmonic content. Although the harmonic content of electric machines is an extensively studied topic, previous publications tended to focus on one type of synchronous machines and often didn't consider higher time-harmonic orders. This work complements the existing theory by providing a more general approach, thereby covering machines and operating points that weren't covered until now. It considers both three-phase and multi-phase machines with an odd number of phases. The winding distribution can either have an integer or a fractional number of slots per pole and per phase and higher time-harmonic content is regarded as well. Note that saturation is neglected. Despite its general validity, the work succeeds at providing one simple equation to determine the machine's time- and spatial-harmonic content. Moreover, the work also extensively discusses the physical causes of the harmonic content. The combination of this general validity, the simple result and the insight in the physics makes that this work is a strong tool to both study harmonic-related phenomena in electric machines and to develop harmonic-based analytical models.
\end{abstract}

\section{INTRODUCTION}

In modern industry the effect of harmonic content in electrical drives is increasingly important. Indeed, electrical motors are often controlled using pulse-width modulated voltagesource inverters, which results in higher time-harmonic orders in the current signal. The rising importance of these harmonics has led to a growing interest for harmonic-related phenomena in electrical machines, such as noise [1], [2] and losses [3], [4]. To better understand these phenomena, it is mandatory to have a good understanding of which time- and spatialharmonic combinations exist in the machine and what their physical cause is.

On the other hand, a variety of studies and applications require fast and accurate simulation of the magnetic field in an electrical machine. In that light a lot of research on Fourier-based models has been done lately [5]-[16]. Such models analytically compute the machine's magnetic field by expressing it as a summation of harmonic components. The computational time of these models can be significantly reduced if the present time- and spatial-harmonic content is known in advance.

\section{A. Literature}

As follows from the above, a preliminary knowledge of which time- and spatial-harmonic combinations exist in an electric machine's magnetic field is of great interest. Moreover, it is particularly useful to have physical insight in the origin of the machine's harmonic content. This is of course not new, harmonic analysis has been one the most important tools to study electrical machines since long before the rise of power electronics and Fourier-based modeling. It is therefore not surprising that there are a lot of publications on the

Corresponding author: B. Hannon (email: Bert.Hannon@UGent.be). topic. However, the great majority of these publications uses harmonic analysis of the magnetic field as a post-processing tool, i.e. the machine's magnetic field is first calculated and then decomposed in its harmonic content to better understand the results of the calculation, e.g. [17], [18]. Evidently, these articles give only little insight in the harmonic content of electric machines in general, let alone the physical cause for those harmonics.

Publications that do present a broader discussion on the harmonic content of electric machines can mainly be classified according to the type of machines that are studied and to whether, or not, they consider higher time-harmonic orders. In the following, some of the most interesting publications are used to sketch the evolution of the literature on harmonic content. Evidently, these publications are only a small portion of the large amount of literature on the topic.

The most basic publications, such as basic textbooks [19], [20], only consider the spatial-harmonic content of threephase machines with an integer $q, q$ being the number of slots per pole and per phase. Usually, these publications study the MagnetoMotive Force (MMF) to state that the spatialharmonic orders $(k)$ have to satisfy:

$$
k=p(6 c+1)
$$

with $p$ the number of pole pairs and $c$ an integer.

However, as early as the 1950's, several authors published on spatial-harmonic content of machines with an arbitrary number of phases and higher time-harmonic orders. Both Kron [21] and White [22] considered such multi-phase machines, Kron also considered higher time-harmonic orders. However, neither Kron nor White presented clear equations to determine which harmonic orders are present in the electric machine. Such equations can be found in some textbooks on electric machines, such as Pyrhönen's book on the design of electric machines [23] and Boldea's work on induction machines [24]. 
None of the latter publications regarded the harmonic content in machines with a fractional $q$.

In 1983 Klingshirn presented a relation between the MMF's spatial-harmonic orders and what he calls orders of sequence sets [25]. The latter are similar to time-harmonic orders. Although Klingshirn does account for machines with more than three phases, his work is restricted to diametrically wound machines. Later Toliyat presented a paper that does consider machines with concentrated windings [26]. However, his study is restricted to three specific cases and he doesn't give a preliminary discussion of the present harmonic orders. Neither Klingshirn nor Toliyat discussed the harmonic orders other than those originating from the machine's stator-current density. Moreover, none of both papers extensively discusses the physical cause of the harmonic orders.

In 2000, Atallah described the current density on the stator using a Fourier series in his paper on rotor losses [27]. Although he only considers the fundamental time-harmonic order, he presents a clear equation for the spatial-harmonic orders. The equation is valid for machines with integer-slot windings and for machines with fractional-slot windings. However, it will be shown in Section IV that Atallah's findings can be made more strict for machines with an even number of slots per period, i.e. for machines with $\frac{N_{s}}{\tau}$ even. Atallah does not discuss the physical causes for the present harmonic orders, neither does he discuss the harmonic content under no-load conditions.

Similar to Atallah, Zhu described the stator's current density in 2004 [28]. He does account for higher time-harmonic content, but he only considers machines with three phases. Moreove, his formula are not generally valid for machines with Fractional-Slot Windings (FSW).

In 2006, Bianchi discussed machines with fractional-slot windings [29], [30]. He is the first to differentiate between machines with $\frac{N_{s}}{\tau}$ even and $\frac{N_{s}}{\tau}$ odd, he also regards fractional-slot single-layer windings. However, the equations he proposes for spatial-harmonic orders can be made more strict. Bianchi doesn't consider higher time-harmonic content.

As recent as 2014 and 2015, Wei [31], Wang [32] and Zhang [33] have presented interesting insights on the harmonic content of electric machines. Wei does consider a preliminary study of the time- and spatial-harmonic content. However, his discussion is limited to machines with an integer number of slots per pole and per phase.

As can be seen from the above, the existing literature does not give a complete overview of the harmonic content in electric machines. Moreover, in most articles the focus is not on the harmonic content as such but on describing the MMF or analyzing the magnetic field. Therefore, this work aims at complementing the existing literature by discussing the entire harmonic spectrum of machines with fractional slots and by differentiating between machines with an even number of slots per period and machines with an odd number of slots per period. Moreover, these equations have to be as generally valid as possible. Specifically, all synchronous machines that are wound using the Star-Of-Slots (SOS) technique, i.e. both machines with integer- and fractional-slot windings, are considered. The work is limited to unsaturated synchronous machines that operate in steady-state, but can be extended to account for saturation and/or asynchronous operation. Apart from providing equations that are very generally valid, this work also aims at giving a clear physical explanation for those equations.

\section{B. Assumptions}

In order to perform the following study a number of assumptions are made. As one of the main goals of this work is to reduce the computational time of Fourier-based models, the following assumptions are limited to those in the majority of publications on analytical models [5]-[16].

A first, and very important, assumption is that the effect of the end windings can be neglected. This is of course only true for machines with a relatively high length to diameter ratio. It is however an assumption that is used by most authors when constructing analytical models.

A second assumption is made with regard to the stator-current density. Only balanced current systems with an odd number of phases are considered. Note that some systems with an even number of phases are eligible as well. Indeed, if the reduced version of such a system is radially symmetrical, the phases can be grouped in an odd number of neighboring phases [23]. The system is then similar to a system with an odd number of phases.

Thirdly, it is assumed that the winding distribution is defined by the SOS. Although some machines are wound using other techniques, the large majority of winding topologies is constructed using this technique.

The effect of saturation on the harmonic content is not regarded in this work. Although saturation occurs in a lot of machines, it is disregarded in most analytical modeling techniques. Finally, it was assumed that the rotor of the studied machine is rotating at synchronous speed. This reduces the validity of this study to synchronous machines that are in a steady state.

The above assumptions are listed as:

- No end-effects

- Balanced system with an odd number of phases

- Winding distribution according to SOS

- No saturation

- Synchronous operation

\section{Paper outline}

The discussion is structured as follows. In Section II the applied reference system is introduced and a basic introduction on the time and spatial dependency of the magnetic field is presented. Sections III-V discus the three aspects that determine the harmonic content of the magnetic field in a PMSM: the permanent magnets, the stator-current density and the machine's geometry. The findings from these sections are summarized in Section VI and general rules for the harmonic combinations under no-load, armature-reaction and load conditions are presented. Section VI also discusses a simple example of how the obtained knowledge can be used. The validity of the presented study for general synchronous machines is discussed in Section VII. Section VIII concludes the paper. Finally, an introduction to the star-of-slots technique 
and its slot groups is given in the Appendices.

Note that Sections III and IV-A present some basic information. However this information is required for the discussion in later sections.

\section{TIME- AND SPATIAL-HARMONIC ORDERS}

To study the time- and spatial-harmonic content of synchronous electrical machines, radial-flux synchronous machines with surface-mounted permanent magnets are considered. In Section VII the presented study is extended to synchronous machines in general.

Figure 1 shows an example of the studied machines. The topology of these machines lends itself to using a cylindrical coordinate system $(r, \phi, z)$ to describe their magnetic field. In the following the spatial coordinate system is fixed to the stator; $(r, \phi, z)$ is thus a stator reference system. However, apart from a spatial dependency, the magnetic field in the machine also has a time $(t)$ dependency.

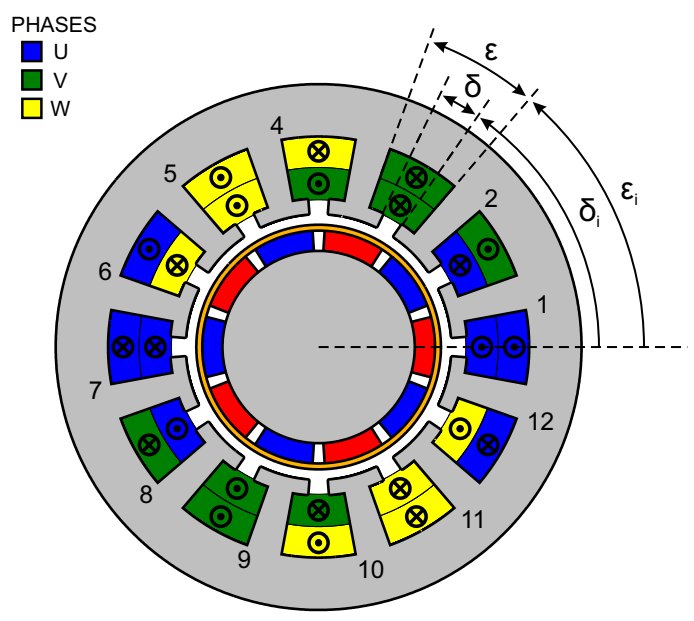

Fig. 1: Geometry of a machine with 12 slots and 5 pole pairs

Obviously the machine's geometry implies a spatial periodicity over $2 \pi$ mechanical radians in the $\phi$-direction. This period is called the basic spatial period. If the machine is operated in steady state, it also has a periodicity over time. This period is called the mechanical time period or the basic time period $\left(T_{m}\right)$, it is the time the rotor needs to perform one revolution. Usually the magnetic field is expressed using auxiliary quantities such as the magnetic scalar potential $(\psi)$ [5]-[7] or the magnetic vector potential (A) [8]-[16]. If the end effects are neglected, $\psi$ and $\mathbf{A}$ are independent of $z$. Moreover, the magnetic vector potential will then only have a $z$-component and thus:

$$
\mathbf{A}=A_{z} \cdot \mathbf{e}_{\mathbf{z}}=A \cdot \mathbf{e}_{\mathbf{z}}
$$

Because of the above mentioned periodicities, $\psi$ and $\mathbf{A}$ can be expressed using the following Fourier series over time and space:

$$
\begin{array}{r}
\psi(r, \phi, t)=\sum_{n=0}^{\infty} \sum_{k=0}^{\infty} \psi_{n, k}^{\cos }(r) \cos \left(k \phi-n \omega_{m} t\right) \\
+\psi_{n, k}^{\sin }(r) \sin \left(k \phi-n \omega_{m} t\right) \\
\begin{aligned}
A(r, \phi, t)=\sum_{n=0}^{\infty} \sum_{k=0}^{\infty} A_{n, k}^{\cos }(r) \cos \left(k \phi-n \omega_{m} t\right) \\
+A_{n, k}^{\sin }(r) \sin \left(k \phi-n \omega_{m} t\right)
\end{aligned}
\end{array}
$$

or in the exponential notation:

$$
\begin{aligned}
& \psi(r, \phi, t)=\sum_{n=-\infty}^{\infty} \sum_{k=-\infty}^{\infty} \psi_{n, k}(r) e^{j\left(k \phi-n \omega_{m} t\right)} \\
& A(r, \phi, t)=\sum_{n=-\infty}^{\infty} \sum_{k=-\infty}^{\infty} A_{n, k}(r) e^{j\left(k \phi-n \omega_{m} t\right)}
\end{aligned}
$$

In equations (3) and (4), $n$ is the time-harmonic order and $k$ is the spatial-harmonic order. The machine's mechanical rotational speed is denoted as $\omega_{m}$.

$$
\omega_{m}=\frac{2 \pi}{T_{m}}
$$

In the rest of this work the magnetic vector potential will be regarded, although a completely similar approach could be used for the magnetic scalar potential. The exponential notation (4b) will be used to reduce the length of the equations. Since the magnetic field can be written as a Fourier series over time and space, every Fourier coefficient $\left(A_{n, k}(r)\right)$ depends on both the time-harmonic order $n$ and the spatial-harmonic order $k$. Therefore $n$ and $k$ should always be regarded together, such time- and spatial-harmonic combination is referred to as $(n, k)$.

The part of the field related to harmonic combination $(n, k)$ is referred to as the $(n, k)$-component of the magnetic field. If $k \phi-n \omega_{m} t$ is assumed constant, the rotational speed of this component can be calculated as:

$$
\frac{\mathrm{d} \phi}{\mathrm{dt}}=\frac{n}{k} \omega_{m}
$$

This means that the rotational speed depends on the $n$ to $k$ ratio. Considering (4), it can easily be seen that both positive and negative rotational speeds are possible.

Although (4) regards every possible harmonic combination $(n, k)$, the magnetic field in a PMSM does not necessarily contain all these combinations. The machine's field, and thereby its harmonic content, is defined by three aspects; the permanent magnets, the distribution of the current density and the machine's geometry. In order to predict the harmonic content of the magnetic field, a good understanding of these aspects is required. Therefore the following sections discuss each of the aforementioned aspects and their impact on the harmonic content of the machine.

\section{Permanent magnets}

The remanent magnetic induction of the permanent magnets $\left(\mathbf{B}_{\text {rem }}\right)$ has a $r$ - and a $\phi$-component. If expressed in a stator 
reference system, $\mathbf{B}_{\text {rem }}$ depends on both space and time and can therefore be written as an exponential Fourier series over space and time:

$$
\begin{aligned}
\mathbf{B}_{\text {rem }}= & B_{r e m, r}(r, \phi, t) \cdot \mathbf{e}_{\mathbf{r}}+B_{r e m, \phi}(r, \phi, t) \cdot \mathbf{e}_{\phi} \\
= & \sum_{n=-\infty}^{\infty} \sum_{k=-\infty}^{\infty} B_{r e m, r, n, k}(r) e^{j\left(k \phi-n \omega_{m} t\right)} \cdot \mathbf{e}_{\mathbf{r}} \\
& +\sum_{n=-\infty}^{\infty} \sum_{k=-\infty}^{\infty} B_{r e m, \phi, n, k}(r) e^{j\left(k \phi-n \omega_{m} t\right)} \cdot \mathbf{e}_{\phi}
\end{aligned}
$$

As a source of magnetic flux, the magnets can, evidently, introduce time- and spatial-harmonic combinations in the machine's magnetic field. They can, however, only introduce combinations that are present in $\mathbf{B}_{\text {rem }}$, i.e. $B_{r e m, r, n, k} \neq 0$ or $B_{r e m, \phi, n, k} \neq 0$. For that reason it is important to understand which harmonic combinations are available in the distribution of the remanent magnetic induction. Note that the fact that the magnets can only introduce harmonic combinations that are present in $\mathbf{B}_{\text {rem }}$ doesn't imply that only these combinations will be present in a no-load situation. As discussed later, the machine's geometry can also introduce harmonic combinations. As mentioned above, it is assumed that the magnets rotate at the mechanical speed $\left(\omega_{m}\right)$, evidently the magnets as such do not change in time. The remanent magnetic induction will thus only contain harmonic combinations that rotate at $\omega_{m}$ mechanical radians per second. Referring to (6), this implies that only harmonic combinations with $n=k$ are eligible.

Secondly, as can be seen in Figure 1, the magnet distribution contains $p$ identical parts along the $\phi$-direction. Where $p$ is the number of pole pairs. These repetitions imply that the spatial period in the $\phi$-direction is $p$ times smaller than the basic spatial period. This smaller period is referred to as the fundamental spatial period and it equals $\frac{2 \pi}{p}$ mechanical radians, which in turn equals $2 \pi$ electrical radians. To comply with the fundamental spatial period, $\mathbf{B}_{\text {rem }}$ can only contain harmonic orders that are a multiple of $p$. Note that this demand requires $p$ identical repetitions of the magnet distribution. If for example one of the magnets is demagnetised, it is no longer valid.

Finally, in most machines the spatial distribution of the remanent magnetic induction is symmetrical in the $\phi$-direction over half a fundamental period. This symmetry can only be maintained if, when referred to the fundamental spatial period, there are no even harmonic orders, i.e $\frac{k}{p}$ is odd. Indeed, as illustrated in Figure 2, even harmonic orders do not show symmetry with respect to the middle of half a period. Note that the demand for odd spatial-harmonic orders is common but not absolute, one could build a machine with asymmetrical magnets.

As a conclusion it can be stated that the magnets will only introduce time-harmonic orders, $n$, wherefore $n \in h_{m}$. With $h_{m}$ the set of time-harmonic orders for which $B_{r e m, r, n, k} \neq 0$ or $B_{r e m, \phi, n, k} \neq 0$. In a healthy machine $h_{m}$ will only contain multiples of $p$. Due to the assumption of synchronous operation the magnets will only introduce harmonic combinations for which $k=n$. If the magnets are symmetrical over half a period, an extra constraint can be imposed: $\frac{k}{p}$ should then be odd.

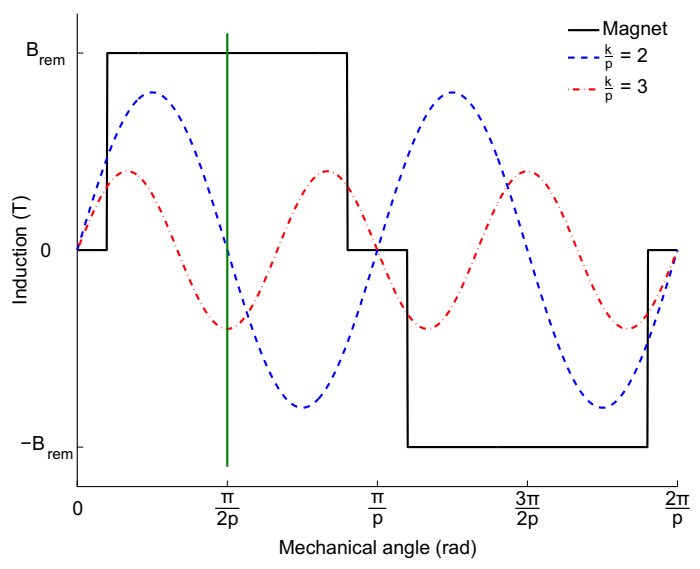

Fig. 2: Illustration of symmetry in the magnet distribution

\section{STATOR-CURRENT DENSITY}

Like the magnets, the current density introduces harmonic combinations. But again, restrictions can be imposed as to which harmonic combinations are induced. In order to study these restrictions, the applied current and the spatial distribution of the windings through which these currents flow are regarded separately.

The time-dependent current will determine which timeharmonic orders are introduced by the current density. The distribution of the windings, in contrast, has a spatial dependency and will determine which spatial-harmonic orders are present.

\section{A. Current system}

The applied current system is a balanced system with an odd number of evenly distributed phases $(m)$. This means that the rotation between neighboring phases is $\frac{2 \pi}{m}$ electrical radians, as illustrated in Figure 3 for a five-phase system.

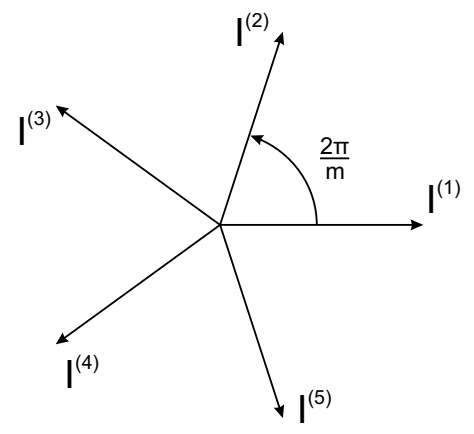

Fig. 3: Balanced five-phase current system

The current, related to an arbitrary phase with number $i_{m} \in$ $[1, m]$, can be written as a Fourier series over time:

$$
I^{\left(i_{m}\right)}=\sum_{n=-\infty}^{\infty} I_{n} e^{-j n\left(\omega_{m} t-\left(i_{m}-1\right) \frac{2 \pi}{m}\right)}
$$

The current density in the machine can only introduce components of the magnetic field whose time-harmonic order 
$n$ corresponds to a non-zero $I_{n}^{(i)}$. The following general consideration on the time-harmonic content of $I^{(i)}$ can be made.

For the sake of uniformity, the mechanical pulsation has been used in (8). However, the base pulsation of current $I^{(i)}$ is the electrical pulsation $\omega_{e}=p \omega_{m}$. This implies that only multiples of $p$ are eligible for $n$. Similar as the fundamental spatial period, the fundamental time period $\left(T_{e}\right)$ can be defined as the base time period $T_{m}$ divided by $p$.

The assumption that the applied system is balanced implies that the sum of the current phasors should always equal zero. When referred to the fundamental time period, this means that the current does not contain time-harmonic orders that are a multiple of $m$. This consideration results in $\frac{n}{p} \neq \mathrm{cm}$, with $c$ an integer.

Often the current waveform is assumed to be symmetrical over half a fundamental time period. If that is the case no even harmonic orders, when referred to the fundamental time period, are present, i.e. $\frac{n}{p}$ is odd.

The above considerations allow stating that the current density will only introduce harmonic combinations with timeharmonic orders that are multiples of $p$ and wherefore $\frac{n}{p}$ is no multiple of $m$. If the current waveform is symmetrical over half a fundamental time period, $\frac{n}{p}$ has to be odd. More generally it can be stated that, if $h_{c}$ contains the time-harmonic orders that are present in $I^{(i)}$, the current density will only introduce time-harmonic orders, wherefore $n \in h_{c}$.

\section{B. Winding distribution}

As mentioned, the distribution of the windings will determine which spatial-harmonic orders are present in the machine's magnetic field. There are a great number of possibilities to distribute the windings around the stator surface. However, mostly the so called star-of-slots technique is used to assign the slots to one or more phases [23], [30], [34], this technique is introduced in Appendix A. Both integer and fractional slot windings can be constructed using this technique. In an attempt to be as general as possible, this work regards all the winding topologies that are feasible using the SOS.

As mentioned, the basics of the SOS theory are discussed in Appendix A. An important parameter related to the SOS is the machine's period $(\tau)$, calculated as the greatest common divider of the amount of pole pairs $(p)$ and the number of slots $\left(N_{s}\right)$.

In Appendix B the term slot group has been defined as a set of adjacent stator slots so that, at synchronous operation, the mechanical shift between similar slots of different slot groups corresponds to the time shift of the current densities linked to these slots. These slot groups are illustrated in Figure 4.

It was shown in Appendix B that, depending on whether $\frac{N_{s}}{\tau}$ is odd or even, the magnetic field will be identical but rotated over $\frac{2 \pi}{m \tau}$ or $\frac{\pi}{m \tau}$ mechanical radians after $\frac{T_{m}}{m \tau}$ or $\frac{T_{m}}{2 m \tau}$ seconds. This can be written mathematically for the magnetic vector potential:

$$
A\left(r, \phi, t_{0}\right)=A\left(r, \phi+\frac{2 \pi}{\varsigma m \tau}, t_{0}+\frac{T_{m}}{\varsigma m \tau}\right)
$$

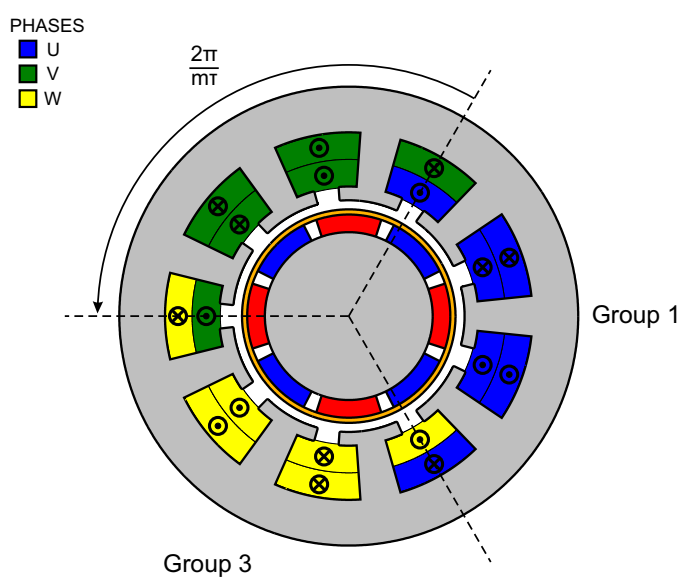

(a) Machine with $\frac{N_{s}}{\tau}$ odd $\left(N_{s}=9, p=4, \tau=1\right)$

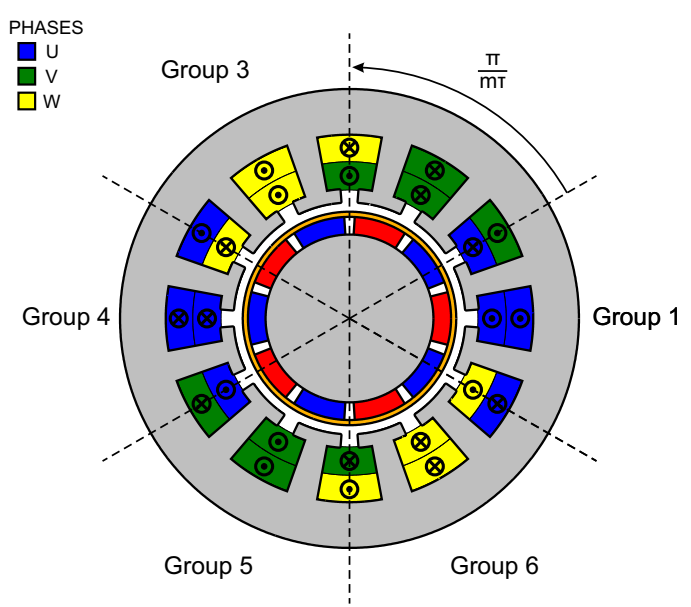

(b) Machine with $\frac{N_{s}}{\tau}$ even $\left(N_{s}=12, p=5, \tau=1\right)$

Fig. 4: Slot groups in electrical machines

where $\varsigma$ is 1 if $\frac{N_{s}}{\tau}$ is odd and 2 if $\frac{N_{s}}{\tau}$ is even.

This time periodicity is not only valid for the complete function, it is also valid for every separate $(n, k)$-component of the magnetic field. Indeed, according to (6) another harmonic component of the magnetic field can only have the same rotational speed if it has both a different time and a different spatial-harmonic order. This, in turn, would imply a different source term, $I_{n^{\prime}}^{(i)}$.

It can thus be written that:

$$
A_{n, k}(r) e^{j\left(k \phi-n \omega_{m} t_{0}\right)}=A_{n, k}(r) e^{j\left(k\left(\phi+\frac{2 \pi}{\varsigma m \tau}\right)-n \omega_{m}\left(t_{0}+\frac{T_{m}}{\varsigma m \tau}\right)\right)}
$$

Knowing that $\omega_{m} T_{m}=2 \pi$, the above can be simplified:

$$
1=e^{j(k-n) \frac{2 \pi}{\varsigma m \tau}}
$$

With $c$ an integer, this results in:

$$
k-n=c \varsigma m \tau
$$

Equation (12) imposes a relation between the time- and spatialharmonic orders. Note that (1) indeed corresponds with (12). More specifically, (1) is the special case where $m=3$ and the machine has an integer amount of slots per pole and per 
phase, which implies $\varsigma=2$ and $\tau=p$. Equation (1) only considers the fundamental time-harmonic order, i.e. $n=p$.

As a conclusion it can be stated that the current density will only introduce time-harmonic orders that are present in the applied currents $\left(n \in h_{c}\right)$. Due to the distribution of the windings, the induced spatial-harmonic orders have to satisfy (12).

\section{MACHINE GEOMETRY}

The third aspect that determines the magnetic field's harmonic spectrum is the geometry. At no-load for example, harmonic combinations, different from the synchronous ones found in Section III, are present in the magnetic field.

The source of these extra harmonic orders is a variation along $\phi$ of the magnetic permeance. This effect is best known as the slotting effect and is mostly associated with slotted machine topologies. The latter is because the amplitude of the induced harmonic components depends on the difference in magnetic permeance, the greater this difference the greater the amplitude. In slotless machines, where the highly permeable teeth are replaced with non-magnetic teeth, the difference between the permeability of the copper windings and that of the non-magnetic teeth is very small. The slotting effect is then so small that most authors neglect it.

As mentioned, the source of the induced spatial-harmonic orders is a difference in magnetic permeance. The reason is that such differences introduce a time periodicity, similar to the one in (9). Indeed, under synchronous operation, the machine's rotor will have rotated over one slot pitch after a time of $\frac{T_{m}}{N_{s}}$ seconds. The rotor will then experience the same stator topology. Under no-load conditions, the magnetic field will then be equal but shifted over one slot pitch. This can be expressed mathematically in terms of the magnetic vector potential:

$$
A\left(r, \phi, t_{0}\right)=A\left(r, \phi+\frac{2 \pi}{N_{s}}, t_{0}+\frac{T_{m}}{N_{s}}\right)
$$

As explained in Section IV, this has to be true for every timeand spatial-harmonic combination separately:

$$
A_{n, k}(r) e^{j\left(k \phi-n \omega_{m} t_{0}\right)}=A_{n, k}(r) e^{j\left(k\left(\phi+\frac{2 \pi}{N_{s}}\right)-n \omega_{m}\left(t_{0}+\frac{T_{m}}{N_{s}}\right)\right)}
$$

And again a relation between the spatial- and time-harmonic orders is found:

$$
k-n=c N_{s}
$$

Where $c$ is an integer. The above mentioned time periodicity is only introduced due to different magnetic permeances in the $\phi$-direction, consequently changes in the $r$-direction do not introduce harmonic combinations.

If the studies machine has teeth with different widths, there will always be a repetition in the shape of the teeth. The righthand side of (15) should then be divided by the number of subsequent teeth after which this set of teeth is repeated. If this number is defined as $N_{t}$, (15) can be rewritten as:

$$
k-n=c \frac{N_{s}}{N_{t}}=c N_{s, e q}
$$

With $N_{s, e q}$ the number of repetitions in the shape of the slots, if the teeth are all equal $N_{t}=1$ and $N_{s, e q}=N_{s}$.

Note that, concerning the armature reaction, the effect of the geometry is embedded in the winding distribution. Therefore the geometry will have no further effect on the harmonic combinations introduced due to the current distribution.

Finally, it should also be noted that differences in the magnetic permeance can also occur on the rotor. However, due to the synchronous rotation of the rotor, these differences do not affect the periodicity found in (13).

As a conclusion, it can be stated that, under no-load conditions, the machine's geometry will introduce harmonic combinations wherefore $k-n=c N_{s, e q}$. However, in a slotless machine, the field components related to combinations for which $c \neq 0$ may be considered negligible.

\section{HARMONIC COMBINATIONS}

In Sections III-V, the harmonic orders introduced due to the magnets, the current density and the geometry were discussed. Based on that discussion it can be concluded that, on the one hand, the source terms, being the permanent magnets and the applied current density, determine which time-harmonic orders will be present. On the other hand, the distribution of the windings and the machine's geometry determine the present spatial-harmonic orders.

Practically, no-load, armature-reaction and load conditions are considered. This section discusses the present harmonic combinations for each of these conditions based on the findings in the previous sections. The section is concluded with a simple example of how the presented results may be used.

\section{A. No load}

Under no-load conditions the currents in the slots equal zero. This means that the harmonic combinations found in Section IV will not be present. The magnets will introduce synchronous harmonic combinations $(n=k)$ that are present in the magnet distribution. For every present time-harmonic order $n$, the geometry will introduce spatial-harmonic orders that satisfy (16). The restriction on the harmonic combinations under no-load conditions can thus be summarized as:

$$
\left\{\begin{array}{l}
n \in h_{m} \\
k-n=c N_{s, e q}
\end{array}\right.
$$

With $c$ an integer and $h_{m}$ the time-harmonic orders for which $B_{r e m, r, n, k} \neq 0$ or $B_{r e m, \phi, n, k} \neq 0$. In a healthy machine $h_{m}$ can only contain time harmonics that are multiples of $p$. If the magnets are symmetrical over half a fundamental period, no time-harmonic orders wherefore $\frac{n}{p}$ is even are present.

\section{B. Armature reaction}

When $\mathbf{B}_{\text {rem }}=\mathbf{0}$, the permanent magnets will not introduce any harmonic combinations. The current density will only introduce the time-harmonic orders that are present in the applied current. The introduced spatial-harmonic orders are defined by the distribution of the current density (12). 
The restrictions on the harmonic combinations can then be summarized as:

$$
\left\{\begin{array}{l}
n \in h_{c} \\
k-n=\operatorname{cs} m \tau
\end{array}\right.
$$

With $c$ an integer and $h_{c}$ the time-harmonic orders for which $I_{n} \neq 0 . h_{c}$ can only contain multiples of $p$ and will not contain any time-harmonic orders for which $\frac{n}{p}$ is a multiple of $m$. If the currents are symmetrical over half a period, no $n$ wherefore $\frac{n}{p}$ is even are present.

\section{Load conditions}

The load situation is a superposition of the no-load and the armature-reaction situations. This implies that all the harmonic combinations that satisfy either (17) or (18) will be present in the magnetic field.

The above is summarized in Table I.

TABLE I: Harmonic content of synchronous electric machines

\begin{tabular}{lll}
\hline Operation & Time-HO (n) & Spatial-HO (k) \\
\hline No load & $n \in h_{m}$ & $k-n=c N_{s, e q}$ \\
Armature reaction & $n \in h_{c}$ & $k-n=c \varsigma m \tau$ \\
Load & $n \in h_{m} \cup h_{c}$ & $k-n=c \varsigma m \tau$ \\
\hline
\end{tabular}

\section{Examples}

To illustrate the applicability of the above theory, two example will be presented in this section. The first, very simple, example shows how Table I can be used to get a better understanding of the machine's physics. Secondly, the above theory was used to construct a Fourier-Based (FB) analytical model of a more complex machine geometry. This model was then validated with Finite-Element (FE) software to confirm the theory's validity. It is shown that applying the above theory results in a huge reduction of the FB model's computational time.

The first example evaluates the effect of an increasing number of slots per pole and per phase on the harmonic content of the no-load field using (17).

If $q$ is increased, the number of slots $\left(N_{s}=2 \mathrm{mpq}\right)$ increases as well. According to (17) this implies fewer spatial-harmonic content. This was indeed expected, increasing the number of slots per pole and per phase is a well-known technique to reduce the harmonic content. Usually only the fundamental time-harmonic order $(n=p)$ is considered. This results in the commonly known spatial-harmonic contents for a machine with three phases and one pole pair:

$$
\begin{cases}k=\cdots,-17,-11,-5,1,7,13,19, \cdots & \text { if } q=1 \\ k=\cdots,-11,1,13, \cdots & \text { if } q=2\end{cases}
$$

However, (17) also allows prediction of the spatial-harmonic content at higher time-harmonic orders. For example if $n=5$, the spatial-harmonic content will be:

$$
\begin{cases}k=\cdots,-19,-13,-7,-1,5,11,17, \cdots & \text { if } q=1 \\ k=\cdots,-19,-7,5,17, \cdots & \text { if } q=2\end{cases}
$$

The above shows that the results presented in this section can lead to a better understanding of the machine's performance. Not only for the fundamental time-harmonic order but also for higher harmonic orders. The presented example is a very simple one, but knowledge of the magnetic field's harmonic combinations may also contribute to the understanding of more complicated physical phenomena like the production of torque and torque ripple.

The second example considers a five-phase, outer-rotor machine with 15 slots and 7 pole pairs, as shown in Figure 5. Note that the yellow cylinder in Figure 5 is a shielding cylinder, i.e. a conductive sleeve wrapped around the magnets to protect them from higher harmonics in the magnetic field. The machine is equipped with a four-layer winding and has a pitch factor of 1. Its parameters are listed in Table II. By means of example, the spatial harmonics corresponding to $n=p=7$, i.e. the fundamental time-harmonic order, and to $n=3 p=21$ can be calculated from Table I. In no-load conditions, the following spatial harmonic orders are obtained:

$$
\begin{cases}k=\cdots,-53,-38,-23,-8,7,22,37,52, \cdots & \text { if } n=7 \\ k=\cdots,-54,-39,-24,-9,6,21,36,51, \cdots & \text { if } n=21\end{cases}
$$

A similar prediction can be made for armature-reaction conditions:

$$
\begin{cases}k=\cdots,-13,-8,-3,2,7,12,17,22, \cdots & \text { if } n=7 \\ k=\cdots,-19,-14,-9,-4,1,6,11,16, \cdots & \text { if } n=21\end{cases}
$$

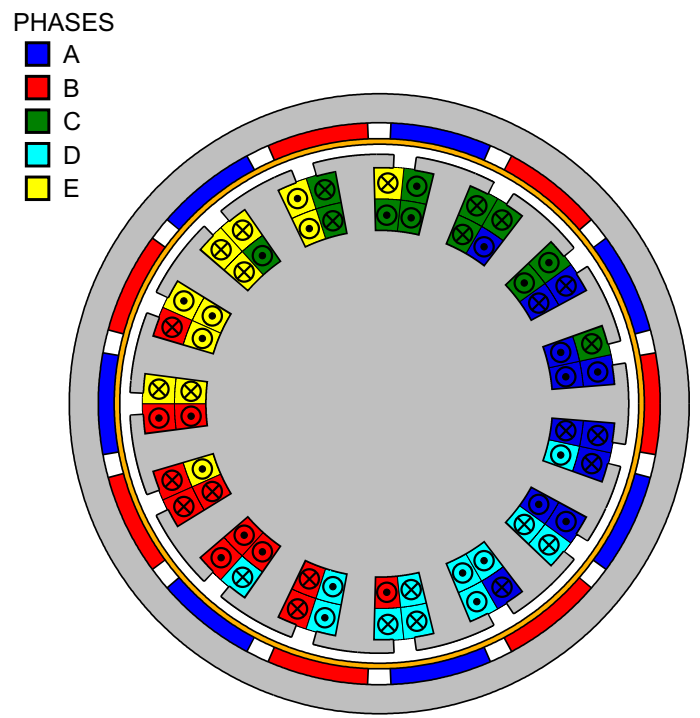

Fig. 5: Geometry of a machine with 5 phases, 15 slots and 7 pole pairs

The above theory was used to construct a computational-time efficient, i.e. only the time- and spatial-harmonic combinations that satisfy Table I are considered, time-dependent Fourierbased analytical model for the 5-phase machine of Figure 5. Assuming a sinusoidal current, the resulting tangential component of the armature-reaction field is compared to results from a FE model in Figure 6. To ensure a good accordance, 
TABLE II: Parameters of the validated five-phase machine

\begin{tabular}{lr}
\hline Parameter & Value \\
\hline Number of slots & 15 \\
Number of pole pairs & 7 \\
Number of phases & 5 \\
Current density in the slots (RMS) & $5 \frac{A}{\mathrm{~mm}^{2}}$ \\
Outer radius of the machine & $79.20 \mathrm{~mm}$ \\
Outer radius of the magnets & $72.20 \mathrm{~mm}$ \\
Outer radius of the shielding cylinder & $66.70 \mathrm{~mm}$ \\
Outer radius of the air gap & $66.70 \mathrm{~mm}$ \\
Outer radius of the teeth tips & $63.70 \mathrm{~mm}$ \\
Outer radius of the teeth & $60.70 \mathrm{~mm}$ \\
Outer radius of the stator yoke & $44.20 \mathrm{~mm}$ \\
Opening angle of the slot openings & $5.54^{\circ}$ \\
Opening angle of the slots & $15.02^{\circ}$ \\
\hline
\end{tabular}

in the periodic subdomains 130 spatial-harmonic orders were considered, in the slots the amount of spatial-harmonic order was set to 15 and the amount of time-harmonic orders was set to 130 as well. The very good accordance between the FE model and the FB model confirms the validity of Table I. Moreover, compared to a traditional time-dependent Fourierbased model, the computational time was reduced from 636.02 seconds to 191.27 seconds. Similar results are obtained for the radial component and for machines in no-load operation. Note that the Fourier-based model has a higher computational time than one might expect from a semi-analytical model, this is due to the fact that it has to consider the field's time-dependency. Indeed, that is the only way to account for the eddy-current reaction-field of the shielding cylinder [13]. Nevertheless, the computational time is still low when compared to that of the time-dependent FE model (2734 s) seconds, which has 42133 degrees of freedom and was run for 8 periods to ensure convergence.

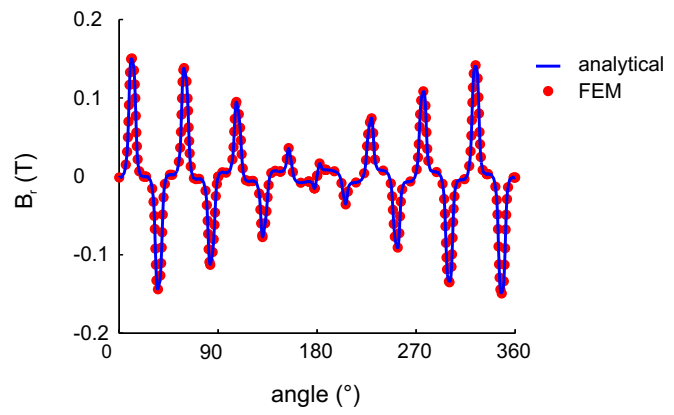

Fig. 6: Tangential component of the magnetic flux density in the center of the air gap at armature-reaction conditions

Note that the FE model was constructed using the same assumptions as those in Section I-B.

\section{VALIDITY}

In the previous sections, a synchronous machine with surface-mounted magnets was considered. However, the results, presented in Section VI, may be applicable more generally. Therefore this section regards the validity of the performed study.

\section{A. Rotor topologies}

As already mentioned in Section $\mathrm{V}$, the differences in magnetic permeance between the magnets and the magnet gaps have no effect on the present harmonic orders. This is true for every difference in magnetic permeance on the rotor. Indeed, such differences do not affect the time periodicity found in (13). This implies that the results from Section VI are also valid for machines with interior magnets.

Despite an increasing popularity of permanent-magnet excited machines, the vast majority of synchronous machines is still excited using electromagnets. Although the excitation source is different in such machines, it has the same characteristics as regards periodicity. If the excitation current is constant, the excitation as such does not vary in time. Combined with the previous consideration that differences in magnetic permeance have no effect, this implies that the obtained results are also valid for machines with a classical excitation.

\section{B. Winding topologies}

The winding topologies considered in Appendices A and B are double-layer topologies. However, the SOS technique can also be used to construct single- or multilayer topologies [30], [34]. Nevertheless, the findings from Section VI are still valid. Indeed, switching to a single or multilayer topology does not change the fact that a number of slot groups can be defined, resulting in a time periodicity as in (9).

Note that for single-layer topologies only half of the slots is considered to construct the star-of-slots [30]. This may affect the number of phase zones.

\section{CONCLUSIONS}

In this work the study of the harmonic content of synchronous electrical machines has been extended to harmonic combinations, where both the time- and spatial-harmonics are regarded. This has resulted in general rules to determine which harmonic combinations are present in the magnetic field, see Section VI. Sections III-V clearly indicate where the different harmonic combination originate from, this information can be used to get a better insight when studying harmonic-related phenomena in synchronous electrical machines. The latter was also illustrated in Section VI-D with a simple example.

The presented work hereby provides an answer to the need for a better understanding of the harmonic content in the magnetic field of synchronous machines. Moreover, some very simple equations to determine the harmonic content of a very broad range of electric machines were presented.

Although the range of machines to which this study applies is very large, induction machines and machines that are not wound according to the star-of-slots theory may contain other harmonic combinations. It would therefore be interesting to perform a similar study for these machines. An other interesting topic for future research is the application of the above findings in Fourier-based models. It is the authors' conviction that the computational time of such models can be drastically reduced by considering the results of this work. 
APPENDIX A

STAR-OF-SLOTS

The so-called Star-Of-Slots (SOS) is a technique that is used to assign the phases of the applied current system to the slots of an electrical machine. It is described by a large number of authors [23], [30], [34] and can be used for both integral and fractional slot windings. N. Bianchi et al. have extended the technique to determine the winding layout of single layer [30] and multilayer [34] topologies. An extensive description of the SOS technique is beyond the scope of this appendix. However, an understanding of the SOS's basics is required for the discussion in Section IV.

In a first step the machine's periodicity $(\tau)$ is calculated as the greatest common divisor of the number of slots and the number of pole pairs:

$$
\tau=\operatorname{gcd}\left(N_{s}, p\right)
$$

Secondly, a system of $\frac{N_{s}}{\tau}$ phasors, called spokes, with a mutual shift of $p \frac{2 \pi}{N_{s}}$ radians is drawn. This is illustrated in Figure 7 for a machine with 12 slots and 5 pole pairs. An example of such a machine is shown in Figure 1. Every spoke now corresponds to a slot in the electrical machine. Logically, the spoke with number $i$ corresponds to the slot with number $i$. The third step is drawing the phase zones. Every phase is assigned two zones, a positive zone and a negative zone. Each phase zone spans $\frac{\pi}{m}$ radians. The shift between two phase zones equals the shift between their corresponding phases, as illustrated in Figure 7 for a three-phase system. Consequently the time shift between the currents linked to the phases of subsequent phase zones is $\frac{1}{\omega_{e}} \frac{\pi}{m}$ seconds.

The resulting diagram defines one conductor of each coil by linking the spokes, and thereby the slots, to the phases of the applied current system. The slot corresponding to the other conductor of the same coil is defined by the coil throw, which is calculated as:

$$
y_{q}=\operatorname{round}\left(\frac{N_{s}}{2 p}\right)
$$

The obtained distribution is repeated after $\frac{N_{s}}{\tau}$ slots.

The winding distribution obtained from the star-of-slots in Figure 7 is shown in Figure 1.

\section{APPENDIX B \\ SLOT GROUPS}

In this section the term slot group is introduced as a number of subsequent slots so that, under synchronous operation, the mechanical shift between two slot groups equals the time shift of their corresponding current densities. In other words, the time the rotor needs to rotate from one slot group to the next equals the time shift of the current densities related to those slot groups.

For simplicity reasons the slot groups are chosen so that each group is dominated by one phase, this is illustrated in Figure 4.

In the following the mechanical shift and the time shift of different slot groups are calculated to prove that they indeed correspond.

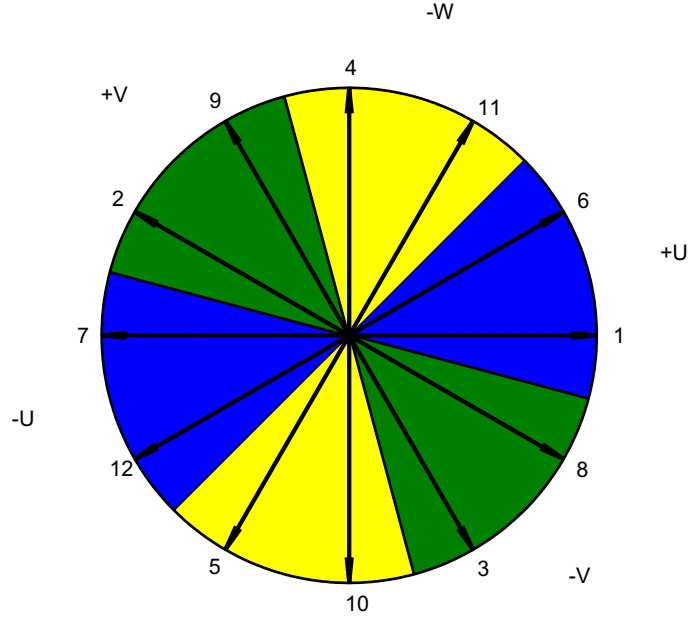

$+\mathrm{W}$

Fig. 7: Star of slots for a machine with $N_{s}=12$ and $p=5$

\section{A. Mechanical shift}

Two cases are regarded; the cases where $\frac{N_{s}}{\tau}$ is odd and the case where $\frac{N_{s}}{\tau}$ is even.

If the number of slots per machine period is odd, every phase will dominate one slot group per machine period. Indeed, every phase should dominate an equal number of similar slot groups, otherwise the winding distribution can never be balanced. A phase dominating more than one slot group, on the other hand, would imply two coinciding spokes in the star of slots. This in turn would imply that all of the following spokes also coincide with another spoke, this can only happen if these spokes belong to another machine period. Therefore a machine with $\frac{N_{s}}{\tau}$ odd contains $m \tau$ slot groups. The number of slots per slot group $\left(N_{g}\right)$ can then be calculated as:

$$
N_{g}=\frac{N_{s}}{m \tau}
$$

The above is illustrated in Figure $4 \mathrm{a}$

If $\frac{N_{s}}{\tau}$ is even, spoke $i+\frac{N_{s}}{2 \tau}$ will be opposite to spoke $i$. Indeed, the rotation between these spokes is:

$$
\frac{N_{s}}{2 \tau} p \frac{2 \pi}{N_{s}}=\frac{p}{\tau} \pi
$$

Since $\frac{N_{s}}{\tau}$ is even and $\tau$ is the greatest common divisor of $N_{s}$ and $p, \frac{p}{\tau}$ has to be odd. This means that spokes $i$ and $i+\frac{N_{s}}{2 \tau}$ are indeed opposite.

Because of the fact that this is true for every spoke, every phase will dominate two similar groups of slots per machine period. One due to the spokes in its positive phase zone and one due to the spokes in its negative phase zone, this can be seen in Figure 4b. This implies that the number of slot groups is now $2 m \tau$, the number of slots in every slot group is then:

$$
N_{g}=\frac{N_{s}}{2 m \tau}
$$

The mechanical shift between similar slots of subsequent slot groups can now be calculated as:

$$
\frac{N_{s}}{\varsigma m \tau} \frac{2 \pi}{N_{s}}=\frac{2 \pi}{\varsigma m \tau}
$$


The mechanical shift, found in (28), translates to a time shift when divided by the synchronous pulsation:

$$
\frac{1}{\omega_{m}} \frac{2 \pi}{\varsigma m \tau}=\frac{T_{m}}{\varsigma m \tau}
$$

\section{B. Time shift}

In the SOS, the angle between two subsequent slots is $p \frac{2 \pi}{N_{s}}$ electrical radians. This implies that in the SOS the angle between similar slots of subsequent slot groups is:

$$
\frac{N_{s}}{\varsigma m \tau} p \frac{2 \pi}{N_{s}}=p \frac{2 \pi}{\varsigma m \tau}
$$

The SOS consists of $2 m$ phase zones with a mutual shift of $\frac{\pi}{m}$ radians, see Appendix A. Knowing this, the number of phase zones between similar slots of subsequent slot groups can be calculated as:

$$
\frac{p \frac{2 \pi}{\varsigma m \tau}}{\frac{\pi}{m}}=\frac{2 p}{\varsigma \tau}
$$

The time shift between currents linked to consecutive phase zones in the SOS is $\frac{\pi}{m}$ electrical radians, see Appendix A. Consequently the shift in electrical radians between the currents linked to similar slots of subsequent slot groups is:

$$
\frac{2 p}{\varsigma \tau} \frac{\pi}{m}
$$

Which results in a time shift when divided by the electrical pulsation:

$$
\frac{2 p}{\varsigma} \frac{\pi}{m \tau} \frac{1}{\omega_{e}}=\frac{T_{m}}{\varsigma m \tau}
$$

This indeed equals (29).

Practically this implies that the rotor will experience the same current density after $\frac{T_{m}}{m \tau}$ or $\frac{T_{m}}{2 m \tau}$ seconds, depending on whether $\frac{N_{s}}{\tau}$ is odd or even. From the stator point of view it means that after $\frac{T_{m}}{m \tau}$ or $\frac{T_{m}}{2 m \tau}$ seconds the armature reaction field will be identical but shifted over $\frac{2 \pi}{m \tau}$ or $\frac{\pi}{m \tau}$ mechanical radians respectively.

\section{REFERENCES}

[1] G. Traxler-Samek, T. Lugand, and M. Uemori, "Vibrational Forces in Salient Pole Synchronous Machines Considering Tooth Ripple Effects," IEEE Transactions on Industrial Electronics, vol. 59, no. 5, pp. 22582266, May 2012.

[2] A. Ruiz-Gonzalez, F. Vargas-Merino, J. Heredia-Larrubia, M. MecoGutierrez, and F. Perez-Hidalgo, "Application of Slope PWM Strategies to Reduce Acoustic Noise Radiated by Inverter-Fed Induction Motors," IEEE Transactions on Industrial Electronics, vol. 60, no. 7, pp. 25552563, Jul. 2013.

[3] T. Mthombeni and P. Pillay, "Lamination core losses in motors with nonsinusoidal excitation with particular reference to PWM and SRM excitation waveforms," IEEE Transactions on Energy Conversion, vol. 20, no. 4, pp. 836-843, Dec. 2005.

[4] P. Sergeant, H. Vansompel, A. Hemeida, A. Bossche, and L. Dupré, "A Computationally Efficient Method to Determine Iron and Magnet Losses in VSI-PWM Fed Axial Flux Permanent Magnet Synchronous Machines," IEEE Transactions on Magnetics, vol. 50, no. 8, pp. 1-10, Aug. 2014.

[5] Z. Q. Zhu, L. J. Wu, and Z. P. Xia, "An Accurate Subdomain Model for Magnetic Field Computation in Slotted Surface-Mounted PermanentMagnet Machines," IEEE Transactions on Magnetics, vol. 46, no. 4, pp. 1100-1115, Apr. 2010.

[6] Z. Zhu, D. Howe, and C. Chan, "Improved analytical model for predicting the magnetic field distribution in brushless permanent-magnet machines," IEEE Transactions on Magnetics, vol. 38, no. 1, pp. 229238, Jan. 2002.
[7] Z. Liu, J. Li, and Q. Jiang, "An improved analytical solution for predicting magnetic forces in permanent magnet motors," Journal of Applied Physics, vol. 103, no. 7, pp. 07F135-07F135-3, Apr. 2008.

[8] A. Bellara, Y. Amara, G. Barakat, and B. Dakyo, "Two-Dimensional Exact Analytical Solution of Armature Reaction Field in Slotted Surface Mounted PM Radial Flux Synchronous Machines," IEEE Transactions on Magnetics, vol. 45, no. 10, pp. 4534-4538, Oct. 2009.

[9] B. L. J. Gysen, K. J. Meessen, J. J. H. Paulides, and E. A. Lomonova, "General Formulation of the Electromagnetic Field Distribution in Machines and Devices Using Fourier Analysis," IEEE Transactions on Magnetics, vol. 46, no. 1, pp. 39-52, Jan. 2010.

[10] T. Lubin, S. Mezani, and A. Rezzoug, "Exact Analytical Method for Magnetic Field Computation in the Air Gap of Cylindrical Electrical Machines Considering Slotting Effects," IEEE Transactions on Magnetics, vol. 46, no. 4, pp. 1092-1099, Apr. 2010.

[11] S. R. Holm, H. Polinder, and J. A. Ferreira, "Analytical Modeling of a Permanent-Magnet Synchronous Machine in a Flywheel," IEEE Transactions on Magnetics, vol. 43, no. 5, pp. 1955-1967, May 2007.

[12] T. Lubin, S. Mezani, and A. Rezzoug, "2-D Exact Analytical Model for Surface-Mounted Permanent-Magnet Motors With Semi-Closed Slots," IEEE Transactions on Magnetics, vol. 47, no. 2, pp. 479-492, Feb. 2011.

[13] B. Hannon, P. Sergeant, and L. Dupré, "2d Analytical Subdomain Model of a Slotted PMSM with Shielding Cylinder," IEEE Transactions on Magnetics, vol. Early Access Online, 2014.

[14] L. J. Wu, Z. Q. Zhu, D. Staton, M. Popescu, and D. Hawkins, "Comparison of analytical models for predicting cogging torque in surface-mounted PM machines," in The XIX International Conference on Electrical Machines - ICEM 2010, Sep. 2010, pp. 1-6.

[15] P. D. Pfister and Y. Perriard, "Slotless Permanent-Magnet Machines: General Analytical Magnetic Field Calculation," IEEE Transactions on Magnetics, vol. 47, no. 6, pp. 1739-1752, Jun. 2011.

[16] P. D. Pfister, X. Yin, and Y. Fang, "Slotted Permanent-Magnet Machines: General Analytical Model of Magnetic Fields, Torque, Eddy Currents, and Permanent-Magnet Power Losses Including the Diffusion Effect,' IEEE Transactions on Magnetics, vol. 52, no. 5, pp. 1-13, May 2016.

[17] K. Komeza and M. Dems, "Finite-Element and Analytical Calculations of No-Load Core Losses in Energy-Saving Induction Motors," IEEE Transactions on Industrial Electronics, vol. 59, no. 7, pp. 2934-2946, Jul. 2012.

[18] Z. Zhu, M. Mohd Jamil, and L. Wu, "Influence of Slot and Pole Number Combinations on Unbalanced Magnetic Force in PM Machines With Diametrically Asymmetric Windings," IEEE Transactions on Industry Applications, vol. 49, no. 1, pp. 19-30, Jan. 2013.

[19] J. Melkebeek and L. Vandevelde, Bouw en Berekening van Elektrische Machines, 5th ed. Ghent, Belgium: Ghent University, 2007.

[20] J. Pollefliet, Electronic Power Control Part 2, 7th ed. Ghent, Belgium: Academia Press, 2012.

[21] G. Kron, Equivalent circuits of electric machinery. New York: John Wiley \& Sons, Ltd, 1951

[22] D. C. White and H. H. Woodson, Electromechanical energy conversion. New York: John Wiley \& Sons, Ltd, 1959.

[23] J. Pyrhönen, J. Tapani, and V. Hrabovcov, Design of Rotating Electrical Machines. Chichester: John Wiley \& Sons, Ltd, Jan. 2008.

[24] I. Boldea and S. A. Nasar, The Induction Machine Handbook. CRC Press, Dec. 2010.

[25] E. Klingshirn, "High Phase Order Induction Motors - Part IIExperimental Results," IEEE Transactions on Power Apparatus and Systems, vol. PAS-102, no. 1, pp. 54-59, Jan. 1983.

[26] H. Toliyat, T. Lipo, and J. White, "Analysis of a concentrated winding induction machine for adjustable speed drive applications. II. Motor design and performance," IEEE Transactions on Energy Conversion, vol. 6, no. 4, pp. 684-692, Dec. 1991.

[27] K. Atallah, D. Howe, P. Mellor, and D. Stone, "Rotor loss in permanentmagnet brushless AC machines," IEEE Transactions on Industry Applications, vol. 36, no. 6, pp. 1612-1618, Nov. 2000.

[28] Z. Zhu, K. Ng, N. Schofield, and D. Howe, "Improved analytical modelling of rotor eddy current loss in brushless machines equipped with surface-mounted permanent magnets," Electric Power Applications, IEE Proceedings -, vol. 151, no. 6, pp. 641-650, Nov. 2004.

[29] N. Bianchi, S. Bolognani, M. Pré, and G. Grezzani, "Design considerations for fractional-slot winding configurations of synchronous machines," IEEE Transactions on Industry Applications, vol. 42, no. 4, pp. 997-1006, Jul. 2006.

[30] N. Bianchi and M. Dai Pré, "Use of the star of slots in designing fractional-slot single-layer synchronous motors," Electric Power Applications, IEE Proceedings -, vol. 153, no. 3, pp. 459-466, May 2006. 
[31] K. Wei, D. Wang, X. Zheng, and S. Cheng, "Research on relationship between harmonic currents and resultant harmonic magnetomotive forces in multiphase machines," in Transportation Electrification Asia-Pacific (ITEC Asia-Pacific), 2014 IEEE Conference and Expo, Aug. 2014, pp. $1-4$.

[32] J. Wang, V. I. Patel, and W. Wang, "Fractional-Slot Permanent Magnet Brushless Machines with Low Space Harmonic Contents," IEEE Transactions on Magnetics, vol. 50, no. 1, pp. 1-9, Jan. 2014.

[33] Z. Zhang, L. Yu, L. Sun, L. Qian, and X. Huang, "Iron Loss Analysis of Doubly Salient Brushless DC Generators," IEEE Transactions on Industrial Electronics, vol. 62, no. 4, pp. 2156-2163, Apr. 2015.

[34] L. Alberti and N. Bianchi, "Theory and Design of Fractional-Slot Multilayer Windings," IEEE Transactions on Industry Applications, vol. 49, no. 2, pp. 841-849, Mar. 2013. 